\title{
Regeneración por semillas en bosques nativos de Nothofagus antarctica bajo uso silvopastoril en Patagonia Sur, Argentina
}

\author{
Seed regeneration in native forests of Nothofagus antarctica under silvopastoral use in \\ Southern Patagonia, Argentina
}

\author{
Héctor A Bahamonde a,b*, Pablo L Peri a,b,c, Lucas H Monelos b , Guillermo Martínez Pastur ${ }^{\text {c,d }}$ \\ *Autor de correspondencia ${ }^{a}$ Instituto Nacional de Tecnología Agropecuaria (INTA), cc 332, (CP 9400) \\ Río Gallegos, Santa Cruz, Argentina, tel./fax: 54-2966-442014, habahamonde@correo.inta.gov.ar \\ ${ }^{\mathrm{b}}$ Universidad Nacional de la Patagonia Austral (UNPA), Argentina. \\ ${ }^{\mathrm{c}}$ Consejo Nacional de Investigaciones Científicas y Técnicas (CONICET), Argentina. \\ ${ }^{\mathrm{d}}$ Centro Austral de Investigaciones Científicas (CADIC), Argentina.
}

\begin{abstract}
SUMMARY
The aim of the present study was to evaluate the effect of silvopastoral use in two pure stands of Nothofagus antarctica (ñire, ñirre) growing at two site qualities on the regeneration cycle from production and quality of seeds, and the establishment and mortality of seedling in Southern Patagonia (Argentina). To evaluate seed production four traps were distributed to capture the spatial variation of each stand during seven years (2004-2010). Also, seed viability and germination were evaluated. In each site, permanent sampling plots were used to measure seedling establishment and mortality. Seed production in stands under silvopastoral use varied from 1 to 40 million seeds ha ${ }^{-1}$, with no differences compared to seed production in surrounding primary forests of the same site quality. Stands under silvopastoral use showed that seed quality varied among sites being higher in the lower site quality. However, there was no difference in seed quality between silvopastoral use and primary forests stands. Seedling establishment was improved in the stands under silvopastoral use compared to primary forests, but mortality rate was high (or total) in all situations. We concluded that silvopastoral use of the studied stands did not modify substantially seed regeneration compared to primary forests.
\end{abstract}

Key words: seed quality, ñire, silvicultural management, seedlings establishment.

\section{RESUMEN}

Los objetivos de este estudio fueron: i) evaluar la regeneración, desde el punto de vista de su producción y calidad de semillas, así como también de la instalación y supervivencia de plántulas, de dos rodales puros de Nothofagus antarctica (ñire, ñirre) bajo uso silvopastoril en distintas calidades de sitio en Patagonia Sur (51 ${ }^{\circ} \mathrm{S}$, Argentina); ii) comparar estos aspectos de la regeneración de ñire entre rodales bajo uso silvopastoril y bosque primario en similares condiciones geográficas y temporales. Para evaluar la producción de semillas, se usaron cuatro trampas de captura distribuidas espacialmente en cada sitio durante siete años (20042010), determinándose además tamaño, viabilidad y germinación. En cada sitio, se instalaron parcelas permanentes de muestreo para cuantificar la incorporación y supervivencia de plántulas. La producción de semillas en los rodales bajo uso silvopastoril varió desde 1 a 40 millones por hectárea, sin diferenciarse de la producción en bosques primarios aledaños de igual calidad de sitio. En los rodales bajo uso silvopastoril la calidad de las semillas varió entre clases de sitio algunos años, siendo mayor en el sitio de calidad inferior. Sin embargo, en general, no se encontraron diferencias entre bosques primarios y con uso silvopastoril. La instalación de plántulas se vio favorecida en los rodales con uso silvopastoril respecto a bosques primarios, no obstante, la supervivencia fue muy baja o nula en todas las situaciones. Se concluyó que en las situaciones estudiadas el uso silvopastoril de los bosques de ñire no modificó sustancialmente su regeneración por semillas respecto a bosques primarios.

Palabras clave: calidad de semillas, ñire, manejo silvícola, instalación de plántulas.

\section{INTRODUCCIÓN}

Los bosques de Nothofagus antarctica (G. Forster) Oerst. (ñire, ñirre) son parte de las masas boscosas subantárticas de Argentina y Chile, encontrándose presentes desde los $36^{\circ} 30^{\prime}$ hasta los $56^{\circ} 00^{\prime} \mathrm{S}$ y desde el nivel del mar hasta los 2.000 m s.n.m. (Veblen et al. 1996). Es una especie con gran capacidad de adaptación a condiciones ambientales extremas, siendo la que alcanza la mayor am- plitud ecológica de los Nothofagus spp. sudamericanos (Donoso et al. 2006). En la Patagonia Sur argentina, existen 144.000 ha de bosque de ñire del tipo arbóreo en la provincia de Santa Cruz (Peri et al. 2009a), de las cuales aproximadamente un $70 \%$ están bajo un uso silvopastoril informal (Peri 2004), con ovinos o bovinos. Este uso se caracteriza principalmente por un pastoreo extensivo combinando el bosque con sectores de estepa y mallines, donde el mismo no se lleva a cabo con un nivel de manejo 
que permita optimizar el uso de estos recursos en un marco sostenible. Por ejemplo, menos del $20 \%$ de los establecimientos hace una separación de ambientes boscosos y pastizales, y menos del $10 \%$ realiza algún tipo de práctica silvícola que tienda a aumentar la productividad del pastizal dentro del bosque (Ormaechea et al. 2009). Cabe destacar, que en el año 2007 se sancionó en Argentina la Ley Nacional 26.331 de "Presupuestos mínimos de Protección Ambiental de los Bosques Nativos", la cual promueve la protección y el uso sostenible del bosque nativo. De acuerdo al ordenamiento territorial de los bosques nativos realizados a exigencias de la Ley mencionada, en la provincia de Santa Cruz se estableció que existen 138.450 ha de ñire con potencial uso silvopastoril bajo planes de manejo (Peri et al. 2009a). Asimismo, en este contexto, existen gran cantidad de antecedentes dedicados al estudio de aspectos ecológicos y productivos de los bosques de ñire bajo uso silvopastoril, por ejemplo: (i) Gallo et al. (2004) informaron un aumento en la diversidad y abundancia de especies de sotobosque, aves e insectos en bosques de ñire con mayor apertura de dosel; (ii) Peri et al. (2008) encontraron una disminución en el retorno potencial de nutrientes en bosques de ñire sometidos a raleo respecto a bosques primarios sin intervención; (iii) Bahamonde et al. (2012a) informaron un aumento en la producción forrajera de gramíneas en lugares más abiertos de bosques de ñire de mejor calidad forestal; (iv) Bahamonde et al. (2012b) reportaron un aumento en la tasa de descomposición de hojarasca en bosques de ñire con mayores aperturas de dosel; y (v) Gargaglione (2011) encontró una mayor absorción de nitrógeno por parte del estrato herbáceo dentro de un bosque de ñire respecto a lugares sin árboles. A partir de estos y otros antecedentes, se han establecido pautas generales de manejo para el uso silvopastoril de estos bosques (Peri et al. 2009b) que incluyen, por ejemplo, la aplicación de raleos que optimicen la productividad del pastizal. Un aspecto clave en el manejo de estos sistemas es la continuidad del estrato arbóreo, basado en la regeneración natural de estos bosques. Sin embargo, los antecedentes relacionados con la dinámica de regeneración de éstos para la Patagonia Sur son escasos, especialmente, bajo un uso silvopastoril, por ejemplo: (i) Tejera et al. (2005) midieron el establecimiento de la regeneración de ñire bajo distintos niveles de cobertura, encontrando un menor establecimiento de plántulas en bosques más densos; y (ii) Soler Esteban et al. (2010) evaluaron la producción de flores y semillas, mientras que Soler Esteban (2012) evaluó la instalación y supervivencia de regeneración en bosques primarios, secundarios y bajo uso silvopastoril de ñire en Tierra del Fuego. En estos últimos trabajos se reportó una interacción en la producción de flores entre años y tratamientos, una mayor producción de semillas en bosques primarios, así como variaciones entre años y tratamientos (bosques primarios, secundarios y bajo uso silvopastoril) en la instalación de regeneración. En otras especies se ha indicado que la reducción del tamaño de un rodal decrece su cantidad y calidad de semillas principalmente por una disminución de la variabilidad genética (Menges 1991).

Otros antecedentes indican que las plántulas de Nothofagus spp. se establecen con mayor éxito en condiciones moderadas de luz y humedad del suelo (Veblen et al. 1996, Martínez Pastur et al. 2011a, 2011b). No obstante, hay otros factores que incidirían en la instalación y supervivencia de la regeneración, tales como la estructura del rodal (Rosenfeld et al. 2006), características del micrositio (Heinemann et al. 2000, Martínez Pastur et al. 2011a) o historia de uso (Alauzis et al. 2004, Gea et al. 2004) como ha sido indicado para bosques de Nothofagus pumilio (Poepp et Endl.) Krasser.

Del mismo modo, Bahamonde et al. (2011) informaron datos de producción y calidad de semillas, así como de regeneración de ñire en bosques primarios creciendo en distintas calidades de sitio en Santa Cruz (Argentina) durante cinco años. Este trabajo describe además la dinámica de regeneración por semillas con énfasis en los principales factores abióticos influyentes y la calidad forestal en la que se desarrollan los bosques de ñire. Sin embargo, aún se desconoce cuales serían los efectos de los raleos en bosques de ñire bajo uso silvopastoril (con el consecuente aumento de la cobertura de gramíneas y de la presión por herbivoría) sobre la dinámica de la regeneración proveniente de semillas, desde la producción y calidad de las mismas hasta la instalación y supervivencia de plántulas. Con base en los antecedentes se hipotetiza que: i) en rodales bajo uso silvopastoril la producción y calidad de semillas, por ende la instalación de plántulas, estarán directamente asociadas a la calidad forestal de los rodales; ii) la disminución del área basal en rodales raleados para su uso silvopastoril se traducirá en una menor producción y calidad de semillas respecto de rodales no intervenidos; iii) la apertura del dosel generará condiciones lumínicas que permitirán un aumento en la instalación de plántulas; $\mathrm{y}$, sin embargo, iv) la supervivencia de las plántulas se verá perjudicada por la competencia ejercida por el estrato herbáceo debido a un aumento de luz en el sotobosque, principalmente en sitios de mejor calidad forestal donde la producción herbácea es mayor.

El objetivo del presente trabajo fue intentar responder las siguientes preguntas: i) $\dot{i}$ Tiene incidencia la calidad de sitio en la regeneración por semillas en rodales de ñire bajo uso silvopastoril? ii) ¿Varía la producción y calidad de las semillas en rodales intervenidos para su uso silvopastoril de ñire respecto a bosques primarios? iii) ¿Se modifica cuantitativamente el establecimiento y supervivencia de plántulas en estos rodales intervenidos? iv) ¿Serán los posibles efectos iguales en rodales desarrollándose en distintas calidades de sitio?

\section{MÉTODOS}

Sitios de muestreo. Se evaluaron dos rodales puros de bosques coetáneos de ñire localizados en la provincia de Santa Cruz creciendo en diferentes calidades de sitio. Estos 
rodales formaban parte de bosques primarios que fueron intervenidos a través de raleos hace aproximadamente 40 años, lo cual permite estudiarlos como ejemplos de bosques intervenidos para uso silvopastoril a largo plazo. En Bahamonde et al. (2011) se presentó la evaluación de la regeneración por semillas de la parte no intervenida de estos rodales (bosques primarios). Los bosques estudiados se localizaron en: (i) Estancia Cancha Carrera correspondiente a clase de sitio IV (IS $_{50}$ entre 3,6 y 5,1 m, altura dominante final entre 8 y $9,9 \mathrm{~m}$ ) en una posición de media

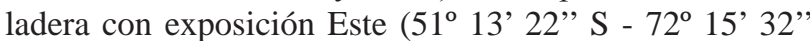
O); y (ii) Estancia Tres Marías un rodal con clase de sitio $\mathrm{V}\left(\mathrm{IS}_{50}<3,6 \mathrm{~m}\right.$, altura dominante final $<8 \mathrm{~m}$ ) siguiendo la clasificación propuesta por Ivancich et al. (2011), este último, ubicado en un terreno ondulado lindando con la estepa ( $51^{\circ} 19^{\prime} 05^{\prime \prime} \mathrm{S}$ - $72^{\circ} 10^{\prime} 47^{\prime \prime}$ O). En la Estancia Cancha Carrera el rodal se encuentra en un potrero que sostiene una carga promedio de 1,6 ovejas madres por hectárea que utilizan el lugar principalmente en invierno, mientras que en la Estancia Tres Marías el rodal recibe cargas variables de ovinos (en duración y categoría animal) sólo en verano (Bahamonde et al. 2011). Se debe aclarar que si bien el ganado puede acceder tanto a los rodales de bosque primario como a los intervenidos para uso silvopastoril, el ingreso a los primeros es mínimo porque son bosques más cerrados $\mathrm{y}$, por ende, con una escaza productividad del estrato herbáceo, lo cual se asocia a la limitación lumínica que se da a mayores coberturas de copa (Bahamonde et al. 2012a). Los rodales evaluados para uso silvopastoril tienen una superficie de 0,4 y 0,6 has en clases de sitio IV y V, respectivamente. Detalles sobre el uso histórico, clima y suelo de los sitios de estudio se presentan en Bahamonde et al. (2011, 2012a).

Caracterización del estrato arbóreo. Cada sitio de muestreo fue caracterizado a partir de tres parcelas circulares de $500 \mathrm{~m}^{2}$ distribuidas al azar, en las cuales se midieron el diámetro normal a 1,3 m (DAP), la altura total (medida con clinómetro y cinta), la cobertura de copas (medida con copímetro de espejo cóncavo; Lemmon 1957). A partir de los datos de las parcelas, se calculó el área basal y la densidad de los rodales. Para el cálculo de volumen de los árboles se usaron las ecuaciones propuestas por Lencinas et al. (2002) para distintas clases de sitio de bosques de ñire. Finalmente, la cobertura del sotobosque fue medida en parcelas de $1 \mathrm{~m}^{2}$ mediante una red de 25 puntos.

Caracterización de las variables ambientales. En cada rodal se midieron en forma continua cada dos horas (entre junio de 2004 y junio de 2010) las temperaturas del aire y del suelo. Los sensores de temperatura del aire se colocaron a una altura de $1 \mathrm{~m}$ del nivel del suelo dentro de una caseta que permite el paso del aire y que además tiene función de protección. La temperatura del suelo se midió mediante termómetros de suelo (HOBO Modelo TMC50-HA, USA) instalados en los primeros $5 \mathrm{~cm}$ del suelo. Con los registros de cada rodal se obtuvieron promedios mensuales. Los datos fueron almacenados mediante data-loggers (HOBO H8 Family, Onset Computer Corporation, USA). La humedad volumétrica del suelo fue medida para cada rodal en sus primeros $30 \mathrm{~cm}$ durante la última semana de noviembre de cada año (2004-2010), a través de un equipo medidor de humedad en suelos Time Domain Reflectometry de precisión comprobada (TDR, marca EIJKELKAMP, Modelo FM-3-14.62, Santa Bárbara, USA).

Producción de semillas. Para cuantificar la producción de semillas se instalaron en cada rodal cuatro trampas de captura de $1 \mathrm{~m}^{2}$ (a $50 \mathrm{~cm}$ de altura sobre el suelo) distribuidas en un gradiente espacial a una distancia de 0,$5 ; 4,2 ; 6,5$ y $9,8 \mathrm{~m}$ desde la base del tronco de los árboles más cercanos en clase de sitio IV y $0,4,5,1,7,1$ y $11,9 \mathrm{~m}$ en el rodal en clase de sitio V. Las trampas estuvieron distanciadas entre sí en un promedio de 22 y $27 \mathrm{~m}$ en clase de sitio IV $\mathrm{y} \mathrm{V}$, respectivamente. Las mismas fueron confeccionadas con tela de media sombra y bastidores de madera que han demostrado ser adecuadas para tal fin (Martínez Pastur et al. 2004). Se colectó el material de las trampas en forma mensual durante el período de caída de semillas para estas latitudes (febrero a mayo) entre los años 2004 y 2010. Las muestras se expresaron en número de semillas por unidad de superficie.

Calidad de las semillas. Las semillas fueron pesadas en balanza analítica $( \pm 0,001 \mathrm{~g})$ para determinar el peso de 1.000 semillas. De cada muestra se seleccionaron 40 semillas para clasificarlas de acuerdo a la presencia del endosperma y embrión en llenas y vacías. Posteriormente, se efectuó un análisis de viabilidad. Las semillas se embebieron en agua estéril durante 48 horas y se les extrajo el pericarpio, realizando la prueba de viabilidad en las semillas llenas. Los contenidos fueron tratados con tri-feniltetrazolium al $1 \%$ durante 48 horas y en oscuridad (Moore 1966). Semillas viables fueron aquellas que presentaron coloración roja en la zona embrionaria debido a un proceso de reducción que tiene lugar en las células vivas.

Para cuantificar el porcentaje de germinación de las semillas se tomaron al azar de tres a cinco muestras de 100 semillas de cada sitio dependiendo de la disponibilidad de semillas de cada año. En primer lugar, se hizo una estratificación de las semillas seleccionadas, manteniéndolas a $4{ }^{\circ} \mathrm{C}$ durante 60 días (Premoli 1991). Posteriormente, las semillas estratificadas fueron puestas en una cámara de germinación según las normas ISTA (1999) para Nothofagus spp., durante un periodo de 60 días. El conteo de plántulas (considerándose como tales aquellas con los dos cotiledones desplegados) se realizó cada dos días.

Regeneración. Para cuantificar la incorporación de plántulas en cada rodal, se instalaron cuatro parcelas permanentes de $1 \mathrm{~m}^{2}$ construidas con marcos de hierro $(1 \mathrm{x} 1 \mathrm{~m}) \mathrm{y}$ subdivididas en cuatro cuadrantes (NE, NO, SO, SE) para 
facilitar la ubicación de cada individuo encontrado en coordenadas X-Y. La mitad de las parcelas permanentes fueron clausuradas con malla de alambre octogonal a fin de aislar el efecto de la ganadería sobre la regeneración. En cada parcela se efectuaron censos al inicio (diciembre) y final (abril) de cada período de crecimiento, midiendo el número de plántulas incorporadas y la tasa de mortalidad correspondiente a cada periodo. Se consideró el mes de diciembre como inicio de instalación de plántulas debido a que observaciones previas en los rodales estudiados indicaron que no existían plántulas de fechas anteriores.

Análisis estadístico. Previo a los análisis de cada situación evaluada se realizaron pruebas exploratorias a fin de verificar el cumplimiento de los supuestos de Normalidad y homogeneidad de varianzas (homocedasticidad) de los datos. Para verificar la normalidad de los datos se realizó la prueba de Shapiro-Wilk $(P \leq 0,05)$, mientras que para verificar la homocedasticidad se utilizó la prueba de Levene $(P \leq 0,05)$. En aquellos casos que no se cumpla alguno de los supuestos se explicitará el análisis alternativo y/o transformación de datos para cumplir con los supuestos.

Los parámetros estructurales, la humedad volumétrica del suelo, la instalación y supervivencia de plántulas en cada rodal fueron evaluados a través de ANDEVA, usándose la prueba de Tukey $(P \leq 0,05)$ para separar medias. A fin de evaluar la producción de semillas y su calidad (peso de 1.000 semillas, proporción de semillas llenas, viables y de germinación) se realizaron ANDEVA con medidas repetidas; siendo la clase de sitio el factor inter-sujetos y los años de muestreo el factor intra-sujetos (Gurevitch y Chester 1986). Para la separación de medias se usó la prueba de Tukey $(P \leq 0,05)$. Para analizar la distribución espacial de la caída de semillas se realizó para cada sitio un ANDEVA con medidas repetidas, siendo la posición (distancia al árbol más cercano) de la trampa de captura el factor inter-sujetos y los años de muestreo el factor intra-sujetos. También se realizaron regresiones con distintos modelos lineales y no lineales entre la proporción de semillas caídas y la distancia al árbol más cercano para encontrar el modelo que mejor represente la distribución espacial de la caída de semillas. Con el fin de determinar si había asociación entre los factores ambientales y la instalación de plántulas se realizaron regresiones lineales simples, usando los factores ambientales medidos (temperatura y humedad del suelo) como variables independientes. Para evaluar la instalación y supervivencia de plántulas entre clases de sitio para un mismo uso (silvopastoril) se utilizó la prueba no paramétrica de Kruskal-Wallis (por falta de normalidad de los datos). Se hicieron análisis de correlación de Spearman entre el porcentaje de supervivencia, y los datos de humedad y temperatura del suelo evaluados previamente. En el caso de humedad del suelo se usaron los valores medidos a fines de noviembre y en el caso de temperatura del suelo se utilizaron los valores del periodo previo a la evaluación de la supervivencia (diciembre-abril).

Con la finalidad de comparar los distintos usos de los rodales evaluados en su producción de semillas, peso de mil semillas y su germinación, se realizaron ANDEVA de medidas repetidas usando la clase de sitio y uso (silvopastoril o bosque primario) como factores intersujetos y los años como factor intrasujetos. Se separaron las medias a través de la prueba de Tukey $(P<0,05)$. A fin de evaluar si existían asociaciones entre la producción de semillas, sus parámetros de calidad, variables ambientales, uso del bosque, clases de sitio y la instalación de regeneración se realizaron análisis de correlación de Spearman entre todas las variables mencionadas. Los datos de los bosques primarios entre los años 2004 y 2008 fueron tomados de Bahamonde et al. (2011), mientras que los datos de los años 2009 y 2010 se presentan en este trabajo a modo informativo (cuadro 7). Para todos los análisis estadísticos se usó el software SPSS 11.5.

\section{RESULTADOS}

Parámetros estructurales y variaciones ambientales. Considerando que los dos rodales no presentaron diferencias en la densidad de árboles, la mejor calidad forestal del rodal en clase de sitio IV se manifestó con valores más altos de área basal y volumen (cuadro 1). Sin embargo, el rodal en clase de sitio $\mathrm{V}$ presentó mayor proporción de árboles co-dominantes y menor porcentaje de árboles suprimidos.

Cuadro 1. Parámetros estructurales de dos rodales muestreados de $N$. antarctica bajo uso silvopastoril desarrollándose en diferentes clases de sitio*.

Main dasometric parameters of Nothofagus antarctica stands under silvopastoral use growing in two different site classes*.

\begin{tabular}{cccccccccccc}
\hline $\begin{array}{c}\text { Clase de } \\
\text { Sitio }\end{array}$ & $\begin{array}{c}\text { HD } \\
(\mathrm{m})\end{array}$ & $\begin{array}{c}\text { Cobertura } \\
\text { arbórea }(\%)\end{array}$ & $\begin{array}{c}\text { Cobertura de } \\
\text { sotobosque }(\%)\end{array}$ & $\begin{array}{c}\mathrm{N} \\
\left(\text { árboles ha }{ }^{-1}\right)\end{array}$ & $\begin{array}{c}\text { DAP } \\
(\mathrm{cm})\end{array}$ & $\begin{array}{c}\text { AB } \\
\left(\mathrm{m}^{2} \mathrm{ha}^{-1}\right)\end{array}$ & $\begin{array}{c}\text { Dom } \\
(\%)\end{array}$ & $\begin{array}{c}\text { Cod } \\
(\%)\end{array}$ & $\begin{array}{c}\text { Int } \\
(\%)\end{array}$ & $\begin{array}{c}\text { Sup } \\
(\%)\end{array}$ & $\begin{array}{c}\text { Vol } \\
\left(\mathrm{m}^{3} \mathrm{ha}^{-1}\right)\end{array}$ \\
\hline IV & $8,0 \mathrm{a}$ & $52 \mathrm{a}$ & $85 \mathrm{a}$ & $418 \mathrm{a}$ & $28,3 \mathrm{a}$ & $47,0 \mathrm{a}$ & $37,0 \mathrm{a}$ & $25,0 \mathrm{~b}$ & $19,0 \mathrm{a}$ & $19 \mathrm{a}$ & $218 \mathrm{a}$ \\
V & $4,7 \mathrm{~b}$ & $50 \mathrm{a}$ & $74 \mathrm{a}$ & $357 \mathrm{a}$ & $25,7 \mathrm{a}$ & $19,8 \mathrm{~b}$ & $43,0 \mathrm{a}$ & $38,0 \mathrm{a}$ & $14,0 \mathrm{a}$ & $5 \mathrm{~b}$ & $78 \mathrm{~b}$ \\
\hline
\end{tabular}

* Clases de sitio de acuerdo a Ivancich et al. (2011). HD: altura media de los árboles dominantes; DAP: diámetro normal a 1,3 m; AB: área basal; Dom: árboles dominantes; Cod: árboles codominantes; Int: árboles intermedios; Sup: árboles suprimidos; Vol: volumen. Letras distintas en una misma columna indican diferencias significativas $(P<0,05)$ entre clases de sitio.

* Site classes based on Ivancich et al. (2011). HD: average height dominant trees, DAP: diameter at breast height, AB: basal area, Dom: dominant trees, Cod: co-dominant trees, Int: intermediate trees, Sup: suppressed trees, Vol: volume. Different letters in the same column indicate significant differences among site classes $(P<0.05)$. 
En general las temperaturas de aire y suelo fueron similares entre sitios, sin embargo hubo algunas variaciones interanuales principalmente en la época invernal (figura 1). En el caso de la humedad volumétrica del suelo no se detectaron diferencias entre sitios ni años.
Producción de semillas. Hubo una variación interanual en la producción de semillas $(P<0,001)$ con valores promedios desde 1,4 a 27,8 millones por hectárea en los años 2004 y 2008, respectivamente (figura 2A). Asimismo, la interacción entre años y clases de sitio fue significativa $(P=0,001)$. Sólo
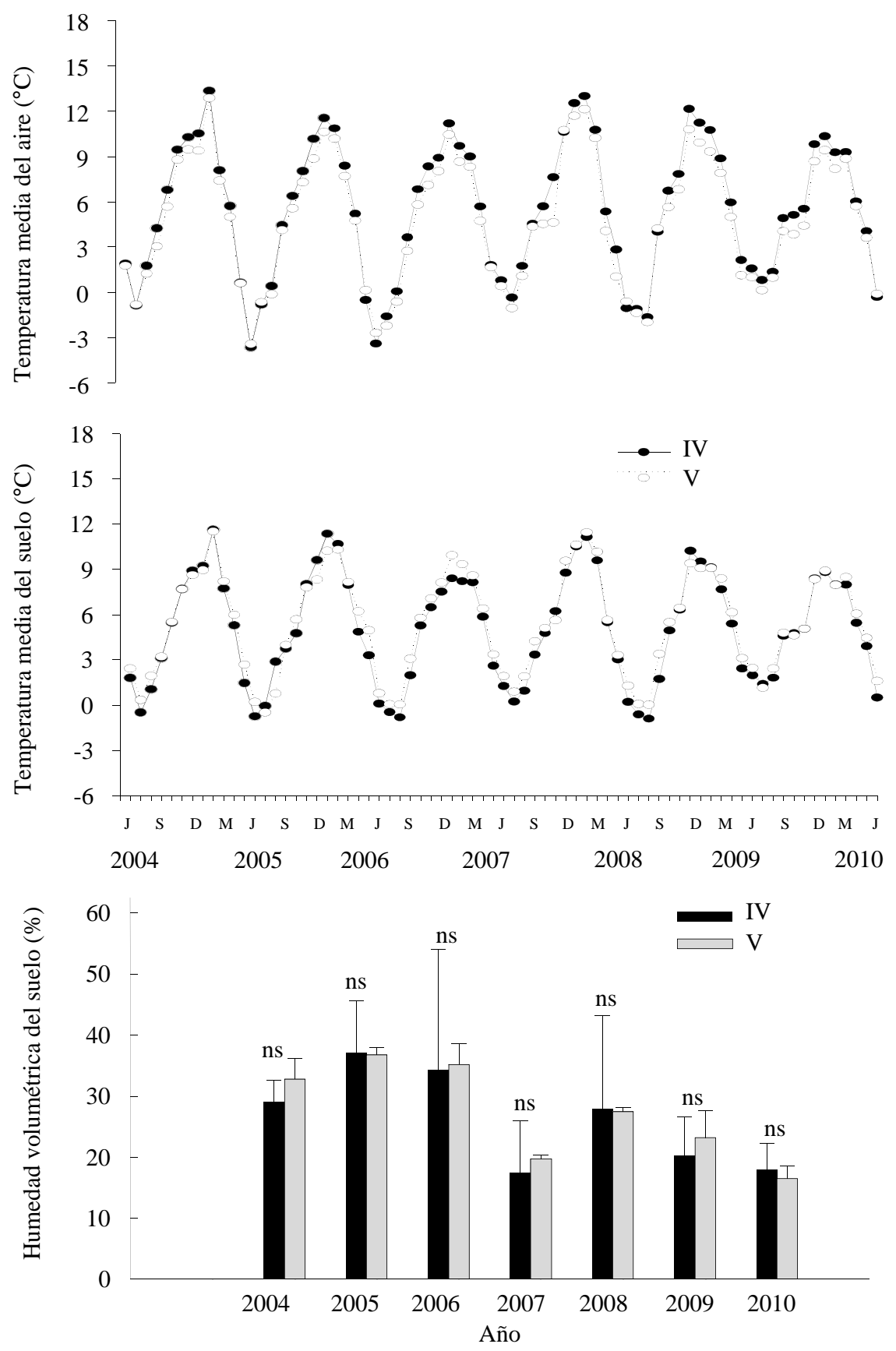

Figura 1. Valores promedios mensuales de temperaturas del aire y del suelo (5 $\mathrm{cm}$ de profundidad) entre junio de 2004 y junio de 2010, y humedad volumétrica del suelo (medidas en la última semana de noviembre de cada año), en bosques de $N$. antarctica bajo uso silvopastoril en dos clases de sitio (IV, V). Las barras representan el desvío estándar de las medias. ns: sin diferencias significativas entre clases de sitio.

Mean monthly values of air and soil (5 cm depth) temperature measured between June 2004 and June 2010, and volumetric soil moisture (measured the last week of November, each year), in Nothofagus antarctica forests under silvopastoral use, growing at two site classes (IV, V). Bars represent standard deviation of the means. ns: not significant differences among sites. 

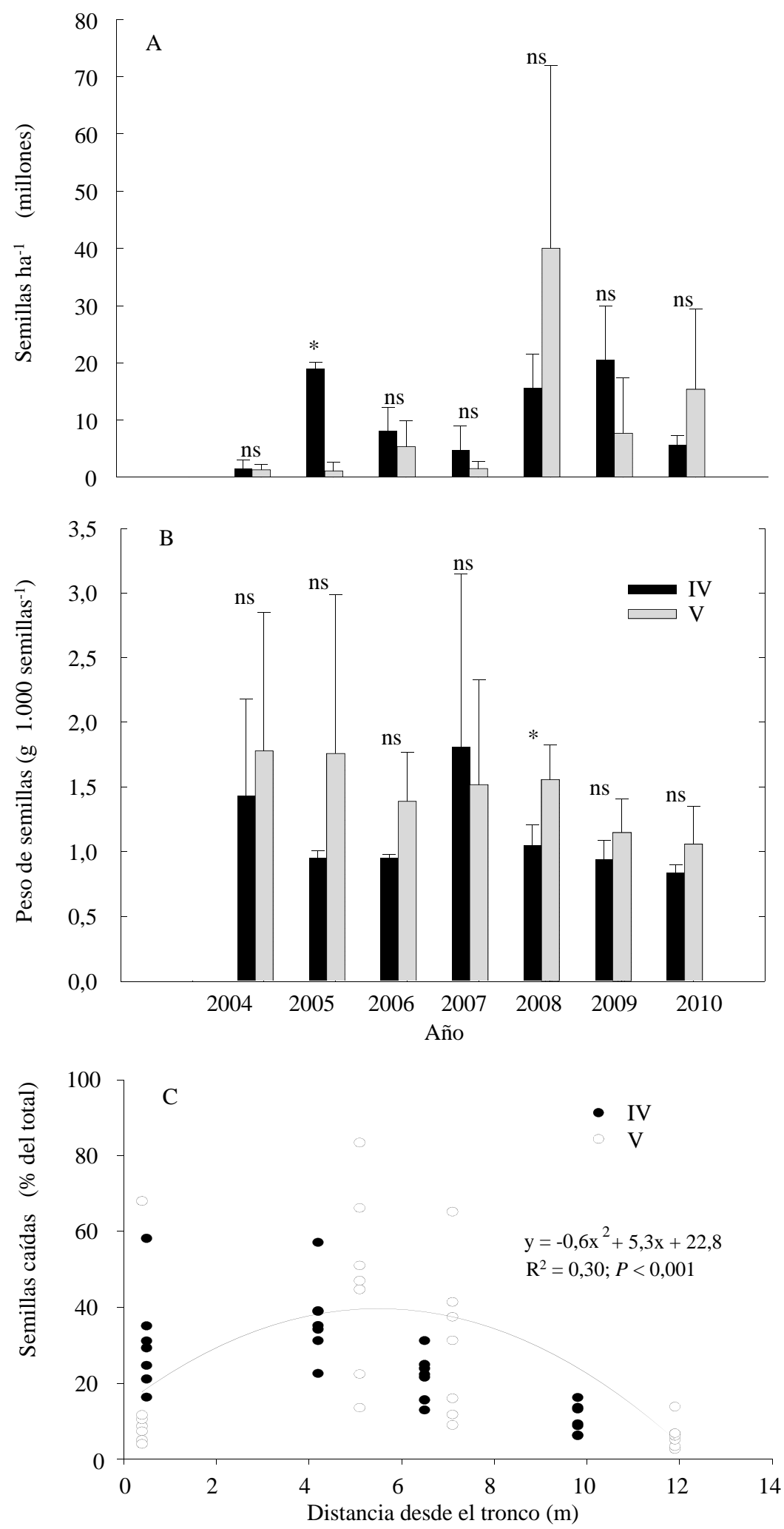

Figura 2. (A) Producción anual de semillas (semillas ha-1); (B) peso de semillas (g 1.000 semillas $^{-1}$ ) y (C) relación entre la distancia al árbol más cercano (m) y semillas caídas (\%) en bosques de $N$. antarctica bajo uso silvopastoril en dos clases de sitio (IV, V) durante siete años (2004-2010). Las barras representan el desvío estándar de las medias. Letras distintas entre clases de sitio para un mismo año indican diferencias estadísticamente significativas: * $P<0,05$, ** $P<0,01$, ns: no significativo.

(A) Annual seed production (seeds ha ${ }^{-1}$ ); (B) seeds weight ( $\left(\mathrm{g} 1,000\right.$ seeds $\left.^{-1}\right)$; and (C) relationship between the distance to nearest tree $(\mathrm{m})$ and fallen seeds (\%) in Nothofagus antarctica forests under silvopastoral use, growing at two site classes (IV, V) during seven years (2004-2010). Bars represent standard deviation of the means. Different letters among site classes for a same year indicate significant differences. ${ }^{*} P<0.05$, ${ }^{* *} P<0.01$, ns: not significant. 
en el año 2005 las diferencias entre clases de sitio fueron significativas, siendo mayor en clase de sitio IV con 19,0 millones por hectárea, mientras que el rodal en clase de sitio $\mathrm{V}$ produjo sólo 1,0 millón de semillas por hectárea (figura 2A).

La distribución espacial de la caída de semillas mostró un patrón similar entre clases de sitio y sin diferencias entre años $(P>0,05)$. Se encontró una relación cuadrática significativa entre la proporción de semillas caídas y la distancia al árbol más cercano (figura 2C). De acuerdo a tal relación la proporción de semillas caídas aumenta con la distancia al árbol más cercano haciéndose máxima a los 4,5 m, disminuyendo linealmente después de esta distancia.

Calidad de semillas. El peso de las semillas no varió significativamente entre años $(P>0,05)$. Solamente en el año 2008 se encontraron diferencias entre clases de sitio $(P<0,05)$ con valores de 1,1 y $1,6 \mathrm{~g} 1000$ semillas $^{-1}$ para las clases de sitio IV y V, respectivamente (figura 2B).

Los porcentajes de semillas llenas variaron entre años $(P=0,001)$, siendo los valores promedios más bajos en 2004 y los más altos en 2009 con 16,5 y 60,0\%, respectivamente. Únicamente se registraron diferencias entre clases de sitio durante el año 2006, donde el rodal en clase de sitio V duplicó el valor de clase de sitio IV (cuadro 2). Similar- mente, la proporción de semillas viables varió entre años $(P<0,001)$ con valores promedio mínimos y máximos de 8,5 y $29,0 \%$ en los años 2009 y 2006 , respectivamente. Diferencias entre sitios se observaron durante los años 2006, 2008 y 2009 con los valores más altos en el rodal correspondiente a clase de sitio V (cuadro 2).

Los porcentajes de germinación de semillas variaron entre años $(P<0,05)$, obteniéndose los valores más altos y bajos en los años 2008 y 2009, respectivamente. Entre clases de sitio se encontraron diferencias, igual que con las semillas viables, durante los años 2006, 2008 y 2009 (cuadro 3 ) siendo más alta la germinación en clase de sitio V.

Regeneración. El establecimiento de plántulas varió entre años $(P<0,05)$ con valores máximos en el año 2008 (cuadro 4) y algunos años sin establecimiento (2005, 2006 y 2010). Por otro lado, no se encontraron diferencias $(P>0,05)$ en el establecimiento de plántulas entre lugares con o sin protección contra la herbivoría. Las diferencias entre clases de sitio dependieron del año, sin embargo, en la mayoría de los casos el establecimiento de plántulas fue mayor en clase de sitio V (cuadro 4). Esta diferencia entre sitios se manifiesta al comparar el promedio de instalación de plántulas del periodo (2004-2010) donde en clases de

Cuadro 2. Porcentajes (media \pm desviación estándar) de semillas llenas y viables de $N$. antarctica bajo uso silvopastoril en dos clases de sitio durante 7 y 6 años de medición, respectivamente.

Sound and viable seeds percentages of Nothofagus antarctica (average \pm standard deviation) under silvopastoral use grown at two site classes during 7 and 6 years of measurements, respectively.

\begin{tabular}{|c|c|c|c|c|c|c|c|}
\hline \multirow{2}{*}{$\begin{array}{l}\text { Clase } \\
\text { de sitio }\end{array}$} & \multicolumn{7}{|c|}{ Semillas llenas } \\
\hline & 2004 & 2005 & 2006 & 2007 & 2008 & 2009 & 2010 \\
\hline IV & $21 \pm 15$ & $50 \pm 14$ & $28 \pm 4$ & $53 \pm 5$ & $33 \pm 3$ & $58 \pm 3$ & $24 \pm 6$ \\
\hline \multirow[t]{3}{*}{$\mathrm{V}$} & $12 \pm 10$ & $35 \pm 1$ & $60 \pm 7$ & $44 \pm 3$ & $56 \pm 1$ & $62 \pm 3$ & $23 \pm 4$ \\
\hline & ns & ns & $*$ & ns & ns & ns & ns \\
\hline & \multicolumn{7}{|c|}{ Semillas viables } \\
\hline IV & - & $15 \pm 0$ & $15 \pm 0$ & $21 \pm 2$ & $12 \pm 1$ & $4 \pm 3$ & $7 \pm 4$ \\
\hline \multirow[t]{2}{*}{$\mathrm{V}$} & - & $10 \pm 7$ & $43 \pm 4$ & $26 \pm 2$ & $32 \pm 2$ & $13 \pm 1$ & $12 \pm 3$ \\
\hline & - & ns & $* * *$ & ns & $* * *$ & $* *$ & ns \\
\hline
\end{tabular}

Significancia estadística entre clases de sitio: $* P<0,05, * * P<0,01$, ns: no significativo.

Statistical significance among site classes: ${ }^{*} P<0.05,{ }^{* *} P<0.01$, ns: not significant.

Cuadro 3. Porcentajes de germinación (media \pm desviación estándar) de semillas de $N$. antarctica bajo uso silvopastoril de dos rodales ubicados en distintas clases de sitio durante siete años de muestreo.

Germination percentages of seeds of Nothofagus antarctica (average \pm standard deviation) under silvopastoral use grown at two site classes during 7 years of measurements.

\begin{tabular}{cccccccc}
\hline Clase de sitio & $\begin{array}{c}2004 \\
(\mathrm{n}=5)\end{array}$ & $\begin{array}{c}2005 \\
(\mathrm{n}=4)\end{array}$ & $\begin{array}{c}2006 \\
(\mathrm{n}=4)\end{array}$ & $\begin{array}{c}2007 \\
(\mathrm{n}=3)\end{array}$ & $\begin{array}{c}2008 \\
(\mathrm{n}=4)\end{array}$ & $\begin{array}{c}2009 \\
(\mathrm{n}=4)\end{array}$ & $\begin{array}{c}2010 \\
(\mathrm{n}=3)\end{array}$ \\
\hline IV & $2,2 \pm 0,3$ & $1,5 \pm 0,7$ & $1,9 \pm 2$ & $9,0 \pm 3,6$ & $2,3 \pm 1,0$ & $1,0 \pm 0,8$ & $4,7 \pm 1,2$ \\
V & $3,1 \pm 0,2$ & $6,1 \pm 3,5$ & $25 \pm 5,8$ & $18,3 \pm 7,8$ & $32,0 \pm 2,1$ & $2,5 \pm 0,6$ & $6,7 \pm 5,5$ \\
& $\mathrm{~ns}$ & $\mathrm{~ns}$ & $* *$ & $\mathrm{~ns}$ & $* * *$ & $*$ & $\mathrm{~ns}$ \\
\hline
\end{tabular}

Significancia estadística entre clases de sitio: $* P<0,05, * * P<0,01$, ns: no significativo.

Statistical significance among site classes: ${ }^{*} P<0.05,{ }^{* *} P<0.01$, ns: not significant. 
Cuadro 4. Establecimiento (E) de plántulas (individuos ha ${ }^{-1}$ ) y supervivencia (S) (\%) de N. antarctica bajo uso silvopastoril de dos rodales ubicados en distintas clases de sitio durante siete ${ }^{\S}$ años de muestreo.

Seedling establishment (E) (seedlings ha ${ }^{-1}$ ) and survival (S) (\%) of Nothofagus antarctica (average \pm standard deviation) under silvopastoral use grown at two site classes during $7^{\S}$ years of measurements.

\begin{tabular}{|c|c|c|c|c|c|c|c|c|c|}
\hline \multirow[t]{2}{*}{ Clase de sitio } & \multirow[t]{2}{*}{ Protección } & \multicolumn{2}{|c|}{$\begin{array}{c}\text { Año } \\
\text { 2004-2005 }\end{array}$} & \multicolumn{2}{|c|}{$\begin{array}{c}\text { Año } \\
2007-2008\end{array}$} & \multicolumn{2}{|c|}{$\begin{array}{c}\text { Año } \\
\text { 2008-2009 }\end{array}$} & \multicolumn{2}{|c|}{$\begin{array}{c}\text { Año } \\
2009-2010\end{array}$} \\
\hline & & $\mathrm{E}$ & S & $\mathrm{E}$ & $\mathrm{S}$ & $\mathrm{E}$ & $\mathrm{S}$ & $\mathrm{E}$ & $\mathrm{S}$ \\
\hline \multirow{2}{*}{ IV } & Con & 20.000 & 0 & 15.000 & 0 & 0 & - & 10.000 & 0 \\
\hline & Sin & 45.000 & 0 & 0 & - & 15.000 & 66,6 & 0 & - \\
\hline \multirow{2}{*}{$\mathrm{V}$} & Con & 80.000 & 0 & 0 & - & 210.000 & 42,5 & 0 & - \\
\hline & Sin & 45.000 & 0 & 0 & - & 270.000 & 35,0 & 40.000 & 75,0 \\
\hline Sig. & & $*$ & ns & $* *$ & ns & $* * *$ & ns & $*$ & $* * *$ \\
\hline
\end{tabular}

Significancia estadística entre clases de sitio (Prueba no paramétrica de Kruskal- Wallis): $* P<0,05$; $* * P<0,01$; *** $P<0,001$; ns: no significativo.

§: Sólo se presentan los datos de los años en que hubo instalación de plántulas.

Statistical significance (Kruskall-Wallis analysis) among site classes: $* P<0.05$, ${ }^{* *} P<0.01$, ${ }^{* * *} P<0.001$, ns: not significant.

s: Only data from years where seedling establishment occurred.

sitio IV y V se midieron valores de 13.000 y 81.000 plántulas ha- ${ }^{-1}$, respectivamente.

El porcentaje de supervivencia durante los años en que hubo establecimiento de plántulas fue variable entre años $(P<0,05)$ y clases de sitio independientemente de la protección contra la herbivoría (cuadro 4). No obstante, en ningún caso las plántulas sobrevivieron un segundo año. No se encontraron asociaciones significativas $(P>0,05)$ entre los factores ambientales evaluados (humedad volumétrica del suelo, temperatura del suelo) y el establecimiento de plántulas en ninguno de los sitios (datos no presentados).

Análisis global de la dinámica de la regeneración. Al analizar los datos de distintos usos (bosques primarios y bajo uso silvopastoril), clases de sitio y años en forma conjunta se pudo observar que la producción de semillas sólo varió $(P<0,001)$ entre años interactuando significativamente con las clases de sitio (cuadro 5). Por ejemplo, en los rodales en clase de sitio IV hubo promedios (entre usos) mínimos y máximos de 1,4 y 30,8 millones de semillas por hectárea en los años 2004 y 2005 , respectivamente; mientras que en clase de sitio $\mathrm{V}$ se registraron valores promedios (entre usos) mínimos y máximos de 1,2 y 40,2 millones de semillas por hectárea en los años 2004 y 2008, respectivamente.

El peso de 1.000 semillas sólo presentó variaciones significativas entre años, sin interactuar con los demás tratamientos, presentando valores promedios (entre clases de sitio y usos) entre 1,0 y $1,8 \mathrm{~g}$ en los años 2010 y 2004, respectivamente. Por otro lado, la germinación de semillas fue significativamente diferente entre usos, clases de sitio y años, encontrándose los valores más altos en el uso silvopastoril, clase de sitio V y en el año 2008. Por ejemplo, promediando años y clases de sitio se obtuvieron valores de 8,2 y $7 \%$ de germinación para uso silvopastoril y bosque primario, respectivamente; al comparar entre clases de sitio los valores promedios fueron de 3 y $12 \%$ en clases de sitio IV y V, respectivamente; mientras la variación interanual presentó valores de entre 2,5 y $20,4 \%$ en los años 2004 y 2008, respectivamente.

Para comparar la instalación de regeneración entre usos (bosque primario o silvopastoril) no fue necesario un análisis estadístico, ya que en bosque primario sólo hubo incorporación de plántulas durante el año 2008 en un solo rodal (Bahamonde et al. 2011).

El establecimiento de plántulas estuvo positivamente asociado a la producción de semillas y la calidad de las mismas (cuadro 6), donde la germinación de semillas se correlacionó positivamente con su tamaño y sanidad. Por otro lado, la producción de semillas no se vio asociada a la cantidad de días con temperatura bajo $0{ }^{\circ} \mathrm{C}$ (DBC) (cuadro 6).

\section{DISCUSIÓN}

La variación interanual en la producción de semillas del presente trabajo se condice con los patrones determinados para bosques primarios de ñire y otras especies del mismo género en Sudamérica (Donoso et al. 2006), y ha sido analizado por Bahamonde et al. (2011). Las diferencias encontradas entre rodales bajo distintas clases de sitio respecto a variables de productividad forestal, como área basal y volumen, no se vieron reflejadas en la producción de semillas (excepto en el año 2005), como si ocurrió en bosques primarios donde las mejores clases de sitio produjeron más semillas (Bahamonde et al. 2011). Los resultados permiten hipotetizar que el rodal que crece en clase de sitio V (menor calidad forestal) produjo mayor cantidad de semillas por árbol, lo cual compensaría su "inferioridad" productiva. Esto tiene sentido si consideramos que el rodal en clase de sitio $\mathrm{V}$ tiene una baja proporción de árboles suprimidos y mayor proporción de árboles codominantes que el rodal en clase de sitio IV. Sin embargo, para poner a prueba esta hipótesis se debiera aumentar el esfuerzo de muestreo considerando la alta variabilidad de los datos durante algunos 
Cuadro 5. ANDEVA de medidas repetidas para la producción, peso y germinación de semillas evaluadas en bosques de Nothofagus antarctica bajo dos usos y dos clases de sitio durante siete años.

Repeated ANOVA measures for seed production, weight and germination of $N$. antarctica, evaluated at different uses ${ }^{\dagger}$ and growing at two site classes ${ }^{\S}$ during 7 years*.

\begin{tabular}{|c|c|c|c|c|}
\hline Fuente & gl & $\begin{array}{l}\text { Producción } \\
\text { de semillas } \\
\text { F }(P)\end{array}$ & $\begin{array}{c}\text { Peso de } 1.000 \\
\text { semillas } \\
\text { F }(P)\end{array}$ & $\begin{array}{c}\text { Germinación } \\
\text { de semillas } \\
\text { F }(P)\end{array}$ \\
\hline \multicolumn{5}{|l|}{ Efectos inter sujetos } \\
\hline $\mathrm{Uso}^{\dagger}$ & 1 & $2,16(0,168)$ & $0,05(0,944)$ & $8,23(0,014)$ \\
\hline Clase de Sitio§(CS) & 1 & $4,69(0,051)$ & $2,71(0,126)$ & $493,14(<0,001)$ \\
\hline \multicolumn{5}{|l|}{ Efectos intra sujetos } \\
\hline Año* & 6 & $21,83(<0,001)$ & $12,04(0,002)$ & $21,89(<0,001)$ \\
\hline \multicolumn{5}{|l|}{ Interacciones } \\
\hline Uso x CS & 1 & $2,09(0,174)$ & $0,01(0,997)$ & $3,46(0,087)$ \\
\hline Uso x Año & 6 & $1,35(0,248)$ & $1,78(0,207)$ & $2,02(0,181)$ \\
\hline CS x Año & 6 & $9,65(<0,001)$ & $0,15(0,704)$ & $27,68(<0,001)$ \\
\hline Uso x CS x Año & 6 & $0,91(0,495)$ & $0,17(0,689)$ & $17,13(0,001)$ \\
\hline
\end{tabular}

${ }^{\dagger}$ Bosque primario y silvopastoril; ${ }^{\S}$ Clases de sitio IV y V; * 2004-2010.

${ }^{\dagger}$ Primary forest and silvopastoral use; ${ }^{\S}$ Site class IV and V; * 2004-2010.

Cuadro 6. Coeficientes de correlación de Spearman (valor $P$ ) entre distintos parámetros de producción y calidad de semillas, regeneración y una variable ambiental, provenientes de bosques de Nothofagus antarctica bajo dos usos (bosque primario y silvopastoril) y dos clases de sitio (IV y V) durante siete años (2004-2010).

Spearman correlation coefficients ( $\mathrm{p}$ value) among different parameters of seed production and quality, regeneration and one environmental variable, in Nothofagus antarctica forests under two uses (primary forest and silvopastoral use) growing at two site classes (IV and V) during seven years (2004-2010).

\begin{tabular}{|c|c|c|c|c|c|c|c|c|c|}
\hline & Cantidad & Tamaño & Germinación & Llenas & Viables & $\mathrm{DBC}$ & Plántulas & Clase de sitio & Uso \\
\hline Cantidad & 1,00 & & & & & & & & \\
\hline Tamaño & $\begin{array}{c}0,10 \\
(0,649)\end{array}$ & 1,00 & & & & & & & \\
\hline Germinación & $\begin{array}{c}0,33 \\
(0,111)\end{array}$ & $\begin{array}{c}0,62 \\
(0,001)\end{array}$ & 1,00 & & & & & & \\
\hline Llenas & $\begin{array}{c}0,43 \\
(0,036)\end{array}$ & $\begin{array}{c}0,53 \\
(0,007)\end{array}$ & $\begin{array}{c}0,64 \\
(<0,001)\end{array}$ & 1,00 & & & & & \\
\hline Viables & $\begin{array}{c}0,39 \\
(0,057)\end{array}$ & $\begin{array}{c}0,54 \\
(0,006)\end{array}$ & $\begin{array}{c}0,88 \\
(<0,001)\end{array}$ & $\begin{array}{c}0,85 \\
(<0,001)\end{array}$ & 1,00 & & & & \\
\hline $\mathrm{DBC}$ & $\begin{array}{c}-0,04 \\
(0,866)\end{array}$ & $\begin{array}{c}0,22 \\
(0,310)\end{array}$ & $\begin{array}{c}0,14 \\
(0,505)\end{array}$ & $\begin{array}{c}0,21 \\
(0,323)\end{array}$ & $\begin{array}{c}0,24 \\
(0,248)\end{array}$ & 1,00 & & & \\
\hline Plántulas & $\begin{array}{c}0,63 \\
(0,001)\end{array}$ & $\begin{array}{c}0,42 \\
(0,042)\end{array}$ & $\begin{array}{c}0,79 \\
(<0,001)\end{array}$ & $\begin{array}{c}0,51 \\
(0,011)\end{array}$ & $\begin{array}{c}0,61 \\
(0,001)\end{array}$ & $\begin{array}{c}-0,01 \\
(0,958)\end{array}$ & 1,00 & & \\
\hline Clase de sitio & $\begin{array}{c}0,22 \\
(0,305)\end{array}$ & $\begin{array}{c}-0,34 \\
(0,103)\end{array}$ & $\begin{array}{c}-0,49 \\
(0,015)\end{array}$ & $\begin{array}{c}-0,10 \\
(0,638)\end{array}$ & $\begin{array}{c}-0,36 \\
(0,079)\end{array}$ & $\begin{array}{c}-0,08 \\
(0,700)\end{array}$ & $\begin{array}{c}-0,29 \\
(0,177)\end{array}$ & 1,00 & \\
\hline Uso & $\begin{array}{c}0,16 \\
(0,455)\end{array}$ & $\begin{array}{c}0,02 \\
(0,908)\end{array}$ & $\begin{array}{c}-0,08 \\
(0,711)\end{array}$ & $\begin{array}{c}-0,17 \\
(0,418)\end{array}$ & $\begin{array}{c}-0,12 \\
(0,585)\end{array}$ & $\begin{array}{c}-0,02 \\
(0,933)\end{array}$ & $\begin{array}{c}-0,06 \\
(0,791)\end{array}$ & $\begin{array}{c}0,00 \\
(>0,999)\end{array}$ & 1,00 \\
\hline
\end{tabular}

DBC: número de días con temperaturas promedio del aire menores a $0{ }^{\circ} \mathrm{C}$ durante junio y agosto del año previo a la caída de semillas.

DBC: days with air temperature under $0{ }^{\circ} \mathrm{C}$ during June and August in the year previous to seeds fall. 
Cuadro 7. Producción y calidad de semillas en dos bosques primarios de N. antarctica creciendo en dos clases de sitio (IV y V), durante dos años (2009-2010).

Seed production and quality in Nothofagus antarctica primary forests growing at two site classes (IV and V), during two years (2009-2010).

\begin{tabular}{cccccc}
\hline $\begin{array}{c}\text { Clase } \\
\text { de sitio }\end{array}$ & $\begin{array}{c}\text { Cantidad } \\
\left(\text { millones ha }^{-1}\right)\end{array}$ & $\begin{array}{c}\text { Peso } \\
\left(\mathrm{g} 1.000 \text { semillas }^{-1}\right)\end{array}$ & $\begin{array}{c}\text { Semillas llenas } \\
(\%)\end{array}$ & $\begin{array}{c}\text { Semillas viables } \\
(\%)\end{array}$ & $\begin{array}{c}\text { Germinación } \\
(\%)\end{array}$ \\
\hline \multicolumn{5}{c}{ Año 2009 } \\
\hline IV & 16,0 & 1,4 & 13,0 & 6,0 & 0,5 \\
V & 6,1 & 1,6 & 33,0 & 24,0 & 13,0 \\
\hline & 14,1 & 0,9 & Año 2010 & \\
\hline IV & 10,7 & 1,3 & 13,0 & 5,0 & 3,0 \\
V & & 17,0 & 10,0 & 3,0 \\
\hline
\end{tabular}

años. Por ejemplo, en el año 2008 la alta variabilidad de la producción de semillas dentro de cada rodal podría estar enmascarando diferencias entre las clases de sitio.

La distribución espacial de la caída de semillas encontrada ha sido previamente documentada para otras especies forestales. Sanguinetti y Kitzberger (2009) indicaron que la mayor concentración de semillas de Araucaria araucana se produjo a $5 \mathrm{~m}$ de árboles semilleros disminuyendo exponencialmente a mayor distancia. Similarmente, Cuevas (2000) informó que la cantidad de semillas producidas por $N$. pumilio disminuye exponencialmente a medida que aumenta la distancia desde los árboles productores. Este patrón espacial de la caída de semillas de ñire está relacionado con sus principales factores de dispersión (viento y gravedad) (Donoso et al. 2006). Los resultados del análisis global incluyendo distintos usos de los bosques de ñire evaluados (bosque primario y uso silvopastoril) sugieren que la remoción de árboles no disminuyó la producción de semillas a nivel rodal como podría esperarse. Contrariamente, Soler Esteban (2012) encontró una asociación directa entre producción de semillas y área basal en rodales de $N$. antarctica con diferentes estructuras forestales en Tierra del Fuego, lo cual se tradujo en que rodales bajo uso silvopastoril produjeron menor cantidad de semillas que bosques primarios y secundarios al referirse los valores de producción de semilla en relación al área basal. En el presente trabajo, estos resultados permiten especular que en los rodales bajo uso silvopastoril se incrementó la producción de semillas por árbol, compensando así la producción de los bosques primarios. Este posible aumento en la producción individual de semillas en sistemas silvopastoriles podría estar dado porque al disminuir el área basal los árboles remanentes reciben luz en mayor cantidad y calidad. En este mismo sentido, considerando que la polinización del ñire es anemófila, la apertura del dosel podría generar condiciones de viento que aumenten la polinización y posterior floración (Williams et al. 2006). Sin embargo, no se pueden descartar otros factores bióticos y abióticos que hayan causado pérdidas de semillas pre o post-dispersión (Donoso 2006, Pulido y Díaz 2005) diferenciales entre rodales o usos (Martínez Pastur et al. 2008), lo cual no fue evaluado en este trabajo. Asimismo, no se puede obviar que los altos valores de dispersión de los datos (especialmente en los rodales bajo uso silvopastoril) podrían ocultar alguna diferencia en la producción de semillas entre usos en alguno de los años evaluados.

El tamaño de las semillas (peso de 1.000 semillas) está dentro del rango informado para la especie en rodales sin intervención silvícola en los mismos sitios evaluados (Bahamonde et al. 2011) a pesar de los cambios generados en los parámetros estructurales (área basal, proporción de clases sociales) de los mismos, así como también en otras latitudes (Premoli 1991). Esto sugiere que el tamaño de las semillas en los sitios evaluados es homogéneo a nivel de individuos. Tampoco hubo diferencias entre clases de sitio en el tamaño de semillas. Esto difiere de otros trabajos donde se han encontrado diferencias asociadas a la estructura forestal o condiciones ambientales en el tamaño de semillas (Donoso 1979, Henríquez 2004). Por otro lado, la variación interanual en el tamaño fue independiente de la variación en la cantidad de semillas producidas por estos rodales, confirmándose la interacción entre sitios y años (Bahamonde et al. 2011).

Similarmente, en los rodales bajo uso silvopastoril, la proporción de semillas llenas y viables se mantuvieron en el rango encontrado en los rodales sin intervención, con gran variación interanual y en algunos años con diferencias entre clases de sitio. Soler Esteban (2012) también encontró variación interanual en viabilidad de semillas de ñire en Tierra del Fuego, aunque con valores más bajos al promedio obtenido en este trabajo. La tendencia, cuando hubo diferencias entre los rodales bajo uso silvopastoril, siempre fue que el rodal en clase de sitio $\mathrm{V}$ presentó valores más altos. En cuanto a los distintos usos, el análisis global indica que no hubo asociación entre distintos usos ni con las semillas llenas ni viables y que la calidad de las semillas en general fue independiente de la cantidad producida. Soler Esteban (2012) encontró una disminución en la viabilidad de semillas de ñire en rodales bajo uso silvopastoril al compararlos con bosques primarios y secundarios, lo cual la autora se lo atribuye a una menor asignación de recursos nutritivos a las estructuras reproductivas, usando como un indicador el menor índice de área foliar, en los rodales sometidos a 
raleos. En los rodales del presente estudio se ha encontrado que efectivamente el retorno potencial de nutrientes, a través de la hojarasca, se ve reducido bajo uso silvopastoril en comparación con bosques primarios (Peri et al. 2008), sin embargo, se ha informado que en estos sitios la apertura del dosel ha aumentado las tasas de descomposición de hojarasca (Bahamonde et al. 2012b), lo cual se traduciría en mayor disponibilidad de nutrientes. Esto podría explicar que no se hayan encontrado diferencias en la producción y calidad de semillas entre distintos usos del bosque.

En general, el potencial de germinación de semillas se mantuvo con valores bajos en comparación a otros Nothofagus spp. sudamericanos (Donoso 2006), y en forma concordante con otros datos bibliográficos para la especie (Premoli 1991). Los valores de germinación siguieron la misma tendencia que la viabilidad con variación interanual y valores más altos en clase de sitio V. El análisis global indica que la proporción de semillas germinadas fue mayor en los rodales bajo uso silvopastoril, sin embargo, la interacción con los años y clases de sitio muestra que tales diferencias estarían dadas por la relativamente alta germinación encontrada algunos años en el rodal clase de sitio V bajo uso silvopastoril, ya que en clase de sitio IV la germinación mostró valores similares entre usos del bosque, lo cual se refleja en las correlaciones encontradas donde la germinación se ve negativamente asociada a la clase de sitio y no asociada al uso del bosque. Si bien no se puede concluir que los rodales bajo uso silvopastoril hayan superado la geminación de bosques primarios, tampoco ocurrió lo contrario como podría esperarse según la bibliografía. Varios trabajos indican que una disminución del área basal de un bosque implica una reducción del poder germinativo de sus semillas debido a una menor variabilidad genética por endogamia (Ellstrand y Elam 1993). Por otro lado, la asociación positiva entre la germinación de las semillas con su proporción de semillas llenas y viables sugiere que los bajos valores de germinación de estos rodales se debería a una limitación en la etapa de fecundación. En este sentido, Donoso (2006) señala la alta auto-incompatibilidad que en general presentan los Nothofagus spp. cuando la variabilidad genética es baja.

El establecimiento de plántulas fue menor a lo reportado para la especie bajo uso silvopastoril en Chubut (Tejera et al. 2005), y dentro del rango de los valores encontrados por Soler Esteban (2012) en Tierra del Fuego. La diferencia en la instalación de plántulas entre clases de sitio en los rodales bajo uso silvopastoril pareciera estar ligada a una combinación de alta producción y germinación de semillas, donde por ejemplo el rodal en clase de sitio $\mathrm{V}$ presentó los mayores valores en los años 2008 y 2009. De manera similar, la baja supervivencia de las plántulas establecidas ha sido también documentada para distintos usos y ubicaciones geográficas de bosques de ñire en Patagonia (Tejera et al. 2005, Bahamonde et al. 2011, Soler Esteban 2012).

$\mathrm{Al}$ analizar entre usos del bosque claramente se vislumbra que la instalación de plántulas fue más exitosa en los rodales bajo uso silvopastoril. Similares resultados en- contraron Tejera et al. (2005) en bosques de ñire creciendo en latitudes más septentrionales. En Tierra del Fuego, Soler Esteban (2012) encontró que durante los años de mayor producción de semillas la instalación de plántulas fue mayor en bosques primarios de ñire, pero en años de baja producción de semillas hubo una incorporación más alta de plántulas en bosques bajo uso silvopastoril. En el presente trabajo, la variación interanual (con algunos años sin instalación de plántulas) estuvo asociada a la cantidad y calidad de semillas producidas. Sin embargo, considerando que ni la cantidad ni la calidad de semillas producidas variaron con los usos de los rodales evaluados y que sólo hubo un año con instalación de plántulas en uno de los rodales de bosque primario (Bahamonde et al. 2011), la instalación de plántulas en estos sitios pareciera estar más vinculada a factores ambientales. En este sentido, la bibliografía señala que, en general, los Nothofagus spp. tienen un establecimiento más exitoso en condiciones moderadamente altas de luz y humedad del suelo (Veblen et al. 1996, Cuevas 2000), y particularmente ñire que sería poco tolerante a la sombra (Frangi et al. 2004). En este estudio los valores de humedad de suelo encontrados en los rodales bajo uso silvopastoril no sería una limitante para el establecimiento de la regeneración. En el caso de los rodales con bosque primario, si bien no se midió la humedad del suelo, la mayor cobertura arbórea disminuiría la cantidad de agua que alcanza el suelo por mayor interceptación y, a su vez, la mayor masa boscosa (en comparación con los rodales bajo uso silvopastoril) con una consecuente mayor evapotranspiración, lo cual hace esperable una menor humedad en el suelo de estos rodales. En este sentido, en los rodales estudiados hay registros entre los años 2003 y 2005 que indican que el agua disponible en el suelo fue menor con coberturas arbóreas mayores (Bahamonde et al. 2012a). Esto sugiere que el mayor éxito relativo de los rodales con uso silvopastoril en la instalación de regeneración estuvo dado principalmente por condiciones de luz y humedad más favorables.

La escasa supervivencia de plántulas durante el primer año y nula supervivencia después del segundo año posteriores a la instalación, concuerdan con los datos obtenidos por Tejera et al. (2005) en distintas coberturas de bosques de ñire en Chubut, y los datos presentados en Bahamonde et al. (2011) para bosques primarios en Santa Cruz. Por el contrario, Soler Esteban (2012) reporta supervivencias similares entre rodales de bosque primario y uso silvopastoril de ñire en Tierra del Fuego con valores promedios de $62 \%$ de supervivencia durante los primeros tres años. Probablemente estas diferencias estén dadas porque los sitios estudiados por Soler Esteban (2012) corresponden a una calidad superior con árboles dominantes de 12,4 $\mathrm{m}$ de altura en los rodales con uso silvopastoril, siendo esto el resultado de una mejor combinación de clima, topografía y suelo (Skovsgaard y Vanclay 2008). Los resultados indican que la supervivencia de las plántulas fue independiente de la presión del ganado ovino por pisoteo o herbivoría, 
descartándose esto como causa única. Existen antecedentes que en los rodales estudiados la humedad del suelo disminuye a medida que transcurre el verano (Bahamonde et al. 2012a). En el mismo trabajo se reportan valores de producción de materia seca de gramíneas dentro del bosque que igualan o superan la producción de lugares adyacentes sin restricción lumínica. Estos datos nos permiten especular que la humedad del suelo y el crecimiento de gramíneas en el sotobosque constituyen restricciones para la supervivencia de la regeneración en estos rodales.

Considerando estos resultados y los antecedentes referidos a la regeneración de ñire, se puede confirmar lo señalado en Bahamonde et al. (2011) respecto al poco éxito de la regeneración por semillas en estos sitios. Asimismo, esto se condice con la bibliografía en el sentido de la importancia que tiene la regeneración vegetativa para ñire (Veblen et al. 1996, Donoso et al. 2006). Sin embargo, la regeneración vegetativa no garantizaría el éxito de la especie ante la presión de la herbivoría ejercida por el ganado en estos sistemas. En este sentido, existen antecedentes en Patagonia Norte que dan cuenta del efecto negativo que ejerce la ganadería (incluso con baja intensidad de carga) sobre la regeneración de especies arbóreas y arbustivas (Raffaele y Veblen 2001). Teniendo en cuenta lo anterior y a fin de garantizar la sustentabilidad del uso de los bosques de ñire como sistemas silvopastoriles, se deben considerar alternativas tendientes a garantizar la continuidad del estrato arbóreo. En esta dirección, en la zona de estudio se están probando protectores individuales de renovales de ñire, con resultados parciales que indican que durante tres años de medición se han alcanzado crecimientos promedio en altura de 10 y $2 \mathrm{~cm}$ año-1 para renovales protegidos y sin protección, respectivamente (Ormaechea y Peri 2010).

\section{CONCLUSIONES}

En general, los datos obtenidos sugieren que la apertura del dosel realizada en estos rodales para su uso silvopastoril no afectó ni la cantidad ni la calidad de semillas producidas. Por el contrario, la apertura del dosel favoreció la instalación de plántulas, sin embargo, los valores de supervivencia fueron escasos o nulos en todas las situaciones al cabo de dos años desde la instalación. No obstante, considerando la alta variabilidad de los datos, para tener conclusiones más certeras respecto al efecto del uso silvopastoril de estos bosques sobre la regeneración por semillas se debiera aumentar el esfuerzo de muestreo tanto a nivel rodal como a escala de paisaje.

\section{AGRADECIMIENTOS}

Se agradece a: Daniel Ledesma, Fernando Coleahue, Alejandro Ojeda, Sabrina Billoni y Javier Szewczuk por su aporte en la recolección y procesamiento de muestras; a Santiago Fernández por permitir la realización de este trabajo en los sitios estudiados.

\section{REFERENCIAS}

Alauzis MV, MJ Mazzarino, E Raffaele, L Roselli. 2004. Wildfires in NW Patagonia: long-term effects on a Nothofagus forest soil. Forest Ecology and Management 192: 131-142.

Bahamonde HA, PL Peri, LH Monelos, G Martínez Pastur. 2011. Aspectos ecológicos de la regeneración por semillas en bosques nativos de Nothofagus antarctica en Patagonia Sur, Argentina. Bosque 32 (1): 20-29.

Bahamonde HA, PL Peri, R Alvarez, A Barneix. 2012a. Producción y calidad de gramíneas en un gradiente de calidades de sitio y coberturas en bosques de Nothofagus antarctica (G. Forster) Oerst. en Patagonia. Ecología Austral 22: 62-73.

Bahamonde HA, PL Peri, R Alvarez, A Barneix, A Moretto, G Martínez Pastur. 2012b. Litter decomposition and nutrients dynamics in Nothofagus antarctica forests under silvopastoral use in Southern Patagonia. Agroforestry Systems 84: 345-360.

Cuevas JG. 2000. Tree recruitment at the Nothofagus pumilio alpine timberline in Tierra del Fuego, Chile. Journal of Ecology 88(5): 840-855.

Donoso C. 1979. Genecological differentiation in Nothofagus obliqua (Mirb.) Oerst. in Chile. Forest Ecology and Management 2: 53-66.

Donoso C. 2006. Las especie arbóreas de los bosques templados de Chile y Argentina. Valdivia, Chile. Marisa Cuneo Ediciones. 678 p.

Donoso C, L Steinke, A Premoli. 2006. Nothofagus antarctica (G. Forster) Oerst. In Donoso Zegers C ed. Las especies arbóreas de los bosques templados de Chile y Argentina. Autoecología. Valdivia, Chile. Marisa Cuneo Ediciones. p. 401-410.

Ellstrand NC, DR Elam. 1993. Population genetic of small population size: implication for plant conservation. Annual Review of Ecology and Systematics 24: 217-242.

Frangi JL, MD Barrera, J Puigdefábregas, PF Yapura, AM Arambarri, L Richter. 2004. Ecología de los bosques de Tierra del Fuego. In Arturi M, J Frangi, JF Goya eds. Ecología y Manejo de los Bosques de Argentina. La Plata, Argentina. Editorial de la Universidad Nacional de La Plata. 88 p.

Gallo E, MV Lencinas, PL Peri. 2004. Desarrollo de sistemas silvopastoriles en bosques de Nothofagus antarctica. Capítulo 2. Biodiversidad en los ñirantales. Informe PIARFON BAP. 24 pp. Consultado 17 Nov. 2011. Disponible en http://www. ambiente.gov.ar/archivos/web/PBVyAP/File/A3/PIARFON\%20BAP/Biodiversidad\%20ire.pdf

Gargaglione V. 2011. Dinámica de macronutrientes en bosques de Nothofagus antarctica de Patagonia Sur. Tesis Doctorado en Ciencias Agrarias. Buenos Aires, Argentina. Escuela para graduados de la Facultad de Agronomía, Universidad de Buenos Aires (UBA). 132 p.

Gea G, G Martínez Pastur, JM Cellini, MV Lencinas. 2004. Forty years of silvicultural management in southern Nothofagus pumilio (Poepp. et Endl.) Krasser primary forests. Forest Ecology and Management 201: 335-347.

Gurevitch J, ST Jr Chester. 1986. Analysis of repeated measures experiments. Ecology 67(1): 251-255.

Heinemann K, T Kitzberger, T Veblen. 2000. Influences of gap microheterogeneity on the regeneration of Nothofagus pumilio in a xeric old-growth forest of northwestern Patagonia, Argentina. Canadian Journal of Forest Research 30(1): 25-31.

Henríquez CA. 2004. Efecto de la fragmentación del hábitat sobre la calidad de las semillas en Lapageria rosea. Revista 
chilena de Historia Natural 77: 177-184.

ISTA (International Rules for Seed Testing). 1999. International Seed Association Rules. Seed Science and Technology 27: 50-52.

Ivancich H, G Martínez Pastur, PL Peri. 2011. Modelos forzados y no forzados para el cálculo de índice de sitio en bosques de Nothofagus antarctica en Patagonia Sur. Bosque 32(2): 135-145.

Lemmon P. 1957. A new instrument for measuring forest overstory density. Journal of Forestry 55: 667-668.

Lencinas MV, G Martínez Pastur, JM Cellini, R Vukasovic, PL Peri, MV Fernández. 2002. Incorporación de la altura dominante y clase de sitio a ecuaciones estándar de volumen para Nothofagus antarctica. (Forster f.) Oersted. Bosque 23(2): 5-17.

Martínez Pastur G, A Saenz, SM Rivera, JM Cellini, A Moretto, MV Lencinas, E Gallo, M Villareal. 2004. Aspectos relacionados con la regeneración del bosque intervenido mediante distintos tipos de corta. Proyecto. Alternativas de Manejo Sustentable para el Manejo Forestal Integral de los bosques de Patagonia. Consultado 16 feb. de 2009. Disponible en http://www.ambiente.gov.ar/archivos/web/PBVyAP/File/ A3/PIARFON\%20BAP/Regeneracion\%20lenga.pdf.

Martínez Pastur G, MV Lencinas, PL Peri, JM Cellini. 2008. Flowering and seeding patterns in unmanaged and managed Nothofagus pumilio forests with a silvicultural variable retention system. Forstarchiv 79: 60-65.

Martínez Pastur G, JM Cellini, MV Lencinas, M Barrera, PL Peri. 2011a. Environmental variables influencing regeneration of Nothofagus pumilio in a system with combined aggregated and dispersed retention. Forest Ecology and Management 261: 178-186.

Martínez Pastur G, PL Peri, JM Cellini, MV Lencinas, M Barrera, H Ivancich. 2011b. Canopy structure analysis for estimating forest regeneration dynamics and growth in Nothofagus pumilio forests. Annal of Forest Science 68: 587-594.

Menges ES. 1991. Seed germination percentage increases with population size in a fragmented prairie species. Conservation Biology 5: 158-164.

Moore RP, 1966. Tetrazolium tests for diagnosing causes for seed weaknesses and for predicting and understanding performance. In Proceedings of the Association of Official Seed Analysts of North America 56. p. 70.

Ormaechea SG, PL Peri, R Molina, JP Mayo. 2009. Situación y manejo actual del sector ganadero en establecimientos con bosque de ñire (Nothofagus antarctica) de Patagonia sur. In Primer Congreso Silvopastoril, Posadas, Misiones, Argentina mayo de 2009. Actas p. 385-393.

Ormaechea SG, PL Peri. 2010. Protección de renovales de ñire al ramoneo en el marco de un uso silvopastoril. In Primeras Jornadas Forestales de Patagonia Sur, Ushuaia, Argentina noviembre de 2010. Actas p. 94.

Peri PL. 2004. Bosque nativo. In González L, Rial P eds. Guía Geográfica Interactiva de Santa Cruz. Buenos Aires, Argentina. Editorial Instituto Nacional de Tecnología Agropecuaria. p. 43-47.

Peri PL, HA Bahamonde, LH Monelos, G Martínez Pastur. 2008. Producción de Hojarasca en Bosques Primarios y bajo Manejo Silvopastoril de Nothofagus antarctica en la Provin- cia de Santa Cruz, Argentina. In Segunda reunión sobre Nothofagus en la Patagonia, EcoNothofagus 2008. Esquel, Argentina. p. 149-155.

Peri PL, E Heinz, M Ferro, P Rial, L Salazar. 2009a. Ordenamiento Territorial de los Bosques Nativos de la provincia de Santa Cruz. 24 pp. Documento elaborado en el marco de la Ley Nacional de No 26331 sobre Presupuestos Mínimos de Protección Ambiental de los Bosques Nativos y Ley Provincial de adhesión $N^{0}$ 3031. Resoluciones del Consejo Agrario Provincial No 166/09 y No 470/09.

Peri PL, N Hansen, V Rusch, L Tejera, L Monelos, M Fertig, H Bahamonde, M Sarasola. 2009b. Pautas de manejo de sistemas silvopastoriles en bosques nativos de Nothofagus antarctica (ñire) en Patagonia. In Actas del Primer Congreso Nacional de Sistemas Silvopastoriles, Posadas, Misiones, Argentina mayo de 2009. Actas. p. 151-155.

Premoli A. 1991. Morfología y capacidad germinativa en poblaciones de Nothofagus antarctica (Forster) Oerst del noroeste andino patagónico. Bosque 12(2): 53-59.

Pulido F, M Díaz. 2005. Regeneration of Mediterranean oak: A whole-cycle approach. Ecoscience 12: 92-102.

Raffaele E, TT Veblen. 2001. Effects of cattle grazing on early postfire regeneration of matorral in northwest Patagonia, Argentina. Natural Areas Journal 21: 243-249.

Rosenfeld JM, RM Navarro Cerrillo, JR Guzman Alvarez. 2006. Regeneration of Nothofagus pumilio (Poep. et Endl) Krasser forest after five years of seed tree cutting. Journal of Environmental Management 78: 44-51.

Sanguinetti J, T Kitzberger. 2009. Efecto de la producción de semillas y la heterogeneidad vegetal sobre la supervivencia de semillas y el patrón espacio-temporal de establecimiento de plántulas en Araucaria araucana. Revista chilena de historia natural 82: 309-335.

Skovsgaard JP, JK Vanklay. 2008. Forest site productivity: a review of the evolution of dendrometric concepts for evenaged stands. Forestry 81:13-31.

Soler Esteban R, G Martínez Pastur, MV Lencinas, PL Peri. 2010. Flowering and seeding patterns in primary, secondary and managed Nothofagus antarctica South Patagonian forests. New Zealand Journal of Botany 48(2): 63-73.

Soler Esteban R. 2012. Regeneración natural de Nothofagus antarctica en bosques primarios, secundarios y bajo manejo silvopastoril. Tesis Doctorado en Ciencias Biológicas. Córdoba, Argentina. Universidad Nacional de Córdoba.126 p.

Tejera L, N Hansen, M Fertig. 2005. Efecto de la cobertura arbórea y del pastoreo vacuno sobre el establecimiento de la regeneración de Nothofagus antarctica (G. Forst.) Oerst. In Actas III Congreso Forestal Argentino y Latinoamericano. Corrientes, Argentina, septiembre de 2005. CD-ROM.

Veblen TT, C Donoso, T Kitzberger, AJ Rebertrus. 1996. Ecology of Southern Chilean and Argentinean Nothofagus forests. In Veblen TT, RS Hill, J Read eds. The ecology and biogeography of Nothofagus forests. London, England. Yale University Press. p. 293-353.

Williams DR, BM Potes, WA Neilsen, KR Joyce. 2006. The effect of tree spacing on the production of flowers in $E u$ calyptus nitens. Australian Forestry 69: 299-304. 
